\title{
Two Refinements of the Chernoff Bound for the Sum of Nonidentical Bernoulli Random Variables
}

\author{
Ye Xia \\ Department of Computer and Information Science and Engineering, University of Florida \\ 301 CSE Building, P.O. Box 116120, Gainesville, Florida 32611-6120, USA
}

\begin{abstract}
We give two refinements to the Chernoff bound for the sum of nonidentical Bernoulli random variables with parameters $p_{i}$, where $0<p_{i}<1$ and $i=1, \ldots, n$. Traditionally, the Chernoff bound is a function of the arithmetic mean of the $p_{i}$ 's, $n^{-1} \sum_{i=1}^{n} p_{i}$. The refined bounds contain the term $n^{-1} \sum_{i=1}^{n} p_{i}^{2}$, and hence, are able to capture the variations of $p_{i}$ 's.
\end{abstract}

Key words: Chernoff bound, Bernoulli Random Variable

\section{Original Chernoff Bound}

Let $X_{1}, X_{2}, \ldots, X_{n}$ be independent Bernoulli random variables, such that $P\left\{X_{i}=\right.$ $1\}=p_{i}$, where $0<p_{i}<1$, for $1 \leq i \leq n$. Let $X=\sum_{i=1}^{n} X_{i}$, and let $p=$ $n^{-1} \sum_{i=1}^{n} p_{i}$. Let $m=(1+\delta) n p$ for $\delta>0$. Then, the Chernoff bound for the sum of the Bernoulli random variables is (Motwani and Raghavan, 1995)

$$
P\{X>m\} \leq\left(\frac{e^{\delta}}{(1+\delta)^{(1+\delta)}}\right)^{n p} .
$$

Note that the Chernoff bound in (1) does not capture the effect of the variation in the parameters, $p_{i}$, where $1 \leq i \leq n$. In this paper, we will find alternative bounds that capture the variation.

Let us define $\nu=n^{-1} \sum_{i=1}^{n} p_{i}^{2}$. Throughout the paper, we assume $n>m$, which is equivalent to $p<1 /(1+\delta)$. The cases where $n \leq m$ are trivial, with $P\{X>$ $m\}=0$.

Email address: yx1@cise.ufl.edu (Ye Xia). 
In all subsequent refinements for the Chernoff bound, the tail probabilities have the form $P\{X>m\}=P\{X>(1+\delta) n p\} \leq \theta^{n}$, where $0<\theta<1$ is dependent of $n$. For the Chernoff bound in (1), $\theta_{C H E R N}=e^{\delta p} /(1+\delta)^{(1+\delta) p}$. One related work is from a special case of Lemma 1 in Chapter X of Petrov (1975). When applied to the Bernoulli random variables, it can be shown that, if $\nu \leq(1-\delta) p$, one can choose $\theta=\exp \left(-\frac{\delta^{2} p^{2}}{2(p-\nu)}\left(1-\frac{\delta p}{2(p-\nu)}\right)\right)$. Due to the restricting condition for this result to hold, we will not explore this result further.

\section{Refinement Using the Inequality $1+x \leq e^{x-a x^{2}}$}

Lemma 2.1 Suppose $0 \leq x \leq u$ for some positive $u$. Then, $1+x \leq e^{x-a x^{2}}$, where $a=(u-\log (1+u)) / u^{2}>0$.

Proof: First, $a>0$ follows from $u>\log (1+u)$ for $u>0$. Next, consider the function $f(x) \triangleq e^{x-a x^{2}}-(1+x)$. Note that $f(0)=f(u)=0$. We claim that $f(x)$ first increases and then decreases on $[0, u]$. Hence, $f(x) \geq 0$ on $[0, u]$.

The first and second derivatives of $f(x)$ are $f^{\prime}(x)=e^{x-a x^{2}}(1-2 a x)-1, f^{\prime \prime}(x)=$ $e^{x-a x^{2}}\left((1-2 a x)^{2}-2 a\right)$. Let us define $g(x)=(1-2 a x)^{2}-2 a$. We will show that, for $u>0, g(0)=1-2 a>0$, and hence, $f^{\prime \prime}(0)>0$. Since $g(0)=u^{-2}\left(u^{2}-2 u+\right.$ $2 \log (1+u))$, it suffices to show that, for $u>0, h(u) \triangleq u^{2}-2 u+2 \log (1+u)>0$. Note that $h(0)=0$ and that, for $u>0, h^{\prime}(u)=2 u^{2} /(1+u)>0$. Then, it must be true that $h(u)>0$ for $u>0$. Otherwise, suppose $h\left(u_{o}\right) \leq 0$ for some $u_{o}>0$. By the Mean-Value theorem, there must exist some $u_{1} \in\left(0, u_{o}\right)$, such that $h^{\prime}\left(u_{1}\right)=\left(h\left(u_{o}\right)-h(0)\right) / u_{o} \leq 0$. With a similar argument, we can show that $f^{\prime}(u)<0$ for $u>0$

We have established that $f^{\prime}(0)=0, f^{\prime \prime}(0)>0$, and for any $u>0, f^{\prime}(u)<0$. In order to satisfy this, $f^{\prime}(x)$ must crosses the $x$-axis an odd number of times on $(0, u]$. We claim that it crosses the $x$-axis only once on $(0, u]$. Suppose it crosses the $x$-axis at least three times. Then, there will be at least three critical points on $[0, u]$. This violates the fact that $f^{\prime \prime}(x)$ has only two zeros on $\mathbb{R}$. As a result, starting at $f^{\prime}(0)=0, f^{\prime}(x)$ first strictly increases above 0 and then strictly decreases below 0 on $[0, u]$. Combining with the fact that $f(u)=0, f(x)$ first increases above 0 and then decreases back to 0 at $x=u$. Hence, $f(x) \geq 0$ for all $x \in[0, u]$.

\section{Theorem 2.2}

$$
P\{X>m\} \leq\left(\theta_{E X P_{-} R E F I N E}\right)^{n}
$$

where

$$
\theta_{E X P_{-} R E F I N E}=\frac{e^{p \delta}}{\left(\frac{(1+\delta) p-\nu}{p-\nu}\right)^{(1+\delta) p-\nu}} .
$$


Proof: For any $t>0, P\{X>m\} \leq e^{-t m} \mathbf{E} e^{t X}=e^{-t m} \prod_{i=1}^{n} \mathbf{E} e^{t X_{i}}$, by the Markov inequality and the independence assumption of the $X_{i}$ 's. The moment generating function for $X_{i}$ is $\mathbf{E} e^{t X_{i}}=1+p_{i}\left(e^{t}-1\right)$. Hence, $P\{X>m\} \leq$ $\prod_{i=1}^{n}\left(1+p_{i}\left(e^{t}-1\right)\right) e^{-t m}$. Applying Lemma 2.1 with $x=p_{i}\left(e^{t}-1\right)$ and $u=e^{t}-1$, we get $a=\left(e^{t}-1-t\right) /\left(e^{t}-1\right)^{2}$, and

$$
\prod_{i=1}^{n}\left(1+p_{i}\left(e^{t}-1\right)\right) e^{-t m} \leq \exp \left(n p\left(e^{t}-1\right)-n \nu\left(e^{t}-1-t\right)-t m\right)
$$

For $p \neq \nu$, the minimum of (4) is achieved at $t^{*}=\log (((1+\delta) p-\nu) /(p-\nu))$, and its value is as the bound in the theorem.

Recall that, throughout the paper, we assume $0<p_{i}<1$ for all $i$. Under this assumption, it is always true that $\nu<p$. It can be shown that the derivative of $\theta_{E X P_{-} R E F I N E}$ with respect to $\nu$ is $\log (1+p \delta /(p-\nu))-p \delta /(p-\nu)$, which is less than zero. Hence, as $\nu$ increases, $\theta_{E X P_{-} R E F I N E}$ decreases. Finally, it can be shown easily that $\theta_{E X P_{-} R E F I N E} \leq \theta_{C H E R N}$. The strength of this refinement over the original Chernoff bound lies in the fact that $e^{x-a x^{2}}$ is a much tighter bound for $1+x$ than $e^{x}$ on $[0, u]$.

\section{Refinement Using a Refined Arithmetic Mean-Geometric Mean Inequality}

Out of various refinements for the arithmetic mean-geometric mean inequality, the following is suitable for our problem.

Theorem 3.1 (Cartwright and Field, 1978) Suppose that $x_{k} \in[\alpha, \beta]$ and $q_{k} \geq 0$ for $k=1, \ldots, n$, where $\alpha>0$, and suppose that $\sum_{k=1}^{n} q_{k}=1$. Then, writing $\bar{x}=\sum_{k=1}^{n} q_{k} x_{k}$, we have

$$
\frac{1}{2 \beta} \sum_{k=1}^{n} q_{k}\left(x_{k}-\bar{x}\right)^{2} \leq \bar{x}-\prod_{k=1}^{n} x_{k}^{q_{k}} \leq \frac{1}{2 \alpha} \sum_{k=1}^{n} q_{k}\left(x_{k}-\bar{x}\right)^{2} .
$$

Define $\sigma^{2}=n^{-1} \sum_{i=1}^{n} p_{i}^{2}-\left(n^{-1} \sum_{k=1}^{n} p_{i}\right)^{2}$. Note that $\sigma^{2} \leq p-p^{2}$.

\section{Theorem 3.2}

$$
P\{X>m\} \leq\left(\theta_{A G \_R E F I N E}\right)^{n},
$$

where

$$
\theta_{A G_{-} R E F I N E}=\left(1+p y_{1}-\frac{\sigma^{2} y_{1}^{2}}{2\left(1+y_{1}\right)}\right)\left(\frac{1}{y_{1}+1}\right)^{(1+\delta) p} .
$$


Here, $y_{1}$ is given by $y_{1}=\left(-a_{1}+\sqrt{a_{1}^{2}-4 a_{0} a_{2}}\right) /\left(2 a_{2}\right)>0$, and $a_{0}, a_{1}$ and $a_{2}$ are given by

$$
\begin{aligned}
& a_{2}=(1-(1+\delta) p)\left(2 p-\sigma^{2}\right) \\
& a_{1}=2\left(p-p \delta-p^{2}-p^{2} \delta-\sigma^{2}\right) \\
& a_{0}=-2 \delta p .
\end{aligned}
$$

Proof: Applying Theorem 3.1 with $x_{k}$ replaced by $1+p_{k}\left(e^{t}-1\right), \beta=e^{t}$ and $q_{k}=1 / n$ for all $k$, we get

$$
\prod_{i=1}^{n}\left(1+p_{i}\left(e^{t}-1\right)\right) e^{-t m} \leq\left(1+p\left(e^{t}-1\right)-\frac{1}{2} e^{-t} \sigma^{2}\left(e^{t}-1\right)^{2}\right)^{n} e^{-t m} .
$$

We will minimize the right hand side of (8). Define $y \triangleq e^{t}-1$, and

$$
\phi(y) \triangleq \log \left(1+p y-\frac{\sigma^{2} y^{2}}{2(1+y)}\right)-(1+\delta) p \log (y+1) .
$$

The right hand side of (8) is equal to $\exp (n \phi(y))$. The derivative of $\phi(y)$ is

$$
\phi^{\prime}(y)=\frac{a_{2} y^{2}+a_{1} y+a_{0}}{(y+1)\left(\left(2 p-\sigma^{2}\right) y^{2}+2(1+p) y+2\right)},
$$

where $a_{0}, a_{1}$ and $a_{2}$ are given as in the statement of the theorem. Let the numerator and denominator of (9) be denoted by $g_{n}(y)$ and $g_{d}(y)$, respectively. It is easy to show that the roots of $g_{d}(y)$ are not greater than 0 , and hence, $\phi^{\prime}(y)$ is continuous on $y>0$. Under the condition $1-(1+\delta) p>0, p>0$ and $\delta>0$, we have $a_{2}>0$ and $-4 a_{0} a_{2}>0$. Hence, the roots of $g_{n}(y)=0$ are real, and the only positive root is $y_{1}=\left(-a_{1}+\sqrt{a_{1}^{2}-4 a_{0} a_{2}}\right) /\left(2 a_{2}\right)>0$. Since $h_{d}(0)=2$ and $h_{d}(y)$ does not have a zero on $y>0$, it must be true $h_{d}(y)>0$ for $y>0$. Therefore, at $y_{1}, \phi^{\prime}(y)$ crosses the $x$-axis increasingly, and hence, $\phi^{\prime \prime}\left(y_{1}\right)>0$. Thus, $y_{1}$ is the minimum of $\phi(y)$ for $y>0$. The right hand side of (8) achieves the minimum on $t>0$ at $t^{*}=\log \left(1+y_{1}\right)$, and the minimum is as the bound in the theorem.

Note that it must be true that $\theta_{A G_{-} R E F I N E} \leq \theta_{A G}$.

\section{References}

D.I. Cartwright and M. J. Field. A Refinement of the Arithmetic Mean-Geometric Mean Inequality. Proceedings of the American Mathematical Society, 71(1):3638, August 1977.

Rajeev Motwani and Prabhakar Raghavan. Randomized Algorithms. Cambridge University Press, 1995.

V. V. Petrov. Sums of Independent Random Variables. Springer Verlag, 1975. 